\title{
MicroRNA-497 inhibits cell proliferation, migration, and invasion by targeting AMOT in human osteosarcoma cells
}

This article was published in the following Dove Press journal:

OncoTargets and Therapy

18 January 2016

Number of times this article has been viewed

\author{
Wen-Dong Ruan \\ Pei Wang \\ Shiqing Feng \\ Yuan Xue \\ Bin Zhang
}

Department of Orthopedics, Tianjin Medical University General Hospital Heping District, Tianjin, People's Republic of China
Correspondence: Wen-Dong Ruan Department of Orthopedics, Tianjin Medical University General Hospital, 154 Anshan Road, Heping District, Tianjin 300052, People's Republic of China

Tel +86 I39 20408055

Email wendongruans@yeah.net
Abstract: MicroRNAs (miRNAs) have a role in the development and progression of human malignancy. The expression of miR-497 is decreased in malignant tumors, which suggests a role for miR-497 as a tumor suppressor. Angiomotin is encoded by the AMOT gene, which is a target for miR-497. Angiomotin has a role in angiogenesis, cell proliferation, and invasion in human malignancies, including osteosarcoma. However, the role of miR-497 in human osteosarcoma is unknown. This preliminary study included human osteosarcoma tissues and normal tissues from 20 patients, the osteosarcoma cell lines, MG-63, SAOS-2, U-2 OS, and the human osteoblast cell line $\mathrm{hFOB}(\mathrm{OB} 3)$. Western blots for angiomotin and quantitative real-time polymerase chain reaction for the expression of miR-497 and AMOT were performed. Knockdown studies were performed using RNA interference and transfection studies used miR-497 mimics. Quantitative cell migration assays were performed, and cell apoptosis was studied by flow cytometry. Osteosarcoma cells and cell lines showed reduced expression of miR-497 and increased expression of angiomotin. Transfection of osteosarcoma cells with miR-497 mimics suppressed the expression of angiomotin. Results from a dual-luciferase reporter system supported AMOT as a direct target gene of miR-497. Knockdown of AMOT using RNA interference resulted in inhibition of osteosarcoma cell proliferation, migration, and invasion. These preliminary studies support a role for miR-497 as a suppressor of AMOT gene expression in human osteosarcoma cells, resulting in suppression of tumor cell proliferation and invasion. Further studies are recommended to investigate the role of miR-497 in osteosarcoma and other malignant mesenchymal tumors.

Keywords: angiomotin, apoptosis, cell migration and invasion, cell proliferation, microRNA-497, osteosarcoma

\section{Introduction}

Osteosarcoma is one of the most common and destructive primary malignant tumors in children and young adults. ${ }^{1}$ It is an aggressive bone tumor characterized by malignant osteoid production and malignant cells with osteoblastic differentiation. ${ }^{2}$ During the past 25 years, the incidence of osteosarcoma has been reported to be increasing at a rate of $1.4 \%$ per year. ${ }^{3}$ Surgery is the standard initial treatment, but even with surgery, the prognosis remains poor. ${ }^{4,5}$ The 5-year survival rate of nonmetastatic osteosarcoma has been improving during the past 40 years to approximately $50 \%-80 \%$; this is due to the introduction of neoadjuvant chemotherapy using doxorubicin, cisplatin, methotrexate, and ifosfamide. However, a poor prognosis remains for patients with recurrent or metastatic osteosarcoma. ${ }^{6,7}$ There are limited established osteosarcomas biomarkers which mean that new and better diagnostic and prognostic molecular targets are required, particularly for patient groups with a high risk of osteosarcoma progression, recurrence, and metastasis. 
MicroRNAs (miRNAs) are a class of small noncoding, endogenous RNAs of between 22 and 24 nucleotides in length that are found in all eukaryotic cells and which play important roles in posttranscriptional regulation. ${ }^{8}$ MiRNAs usually have imperfect binding to the complementary target messenger RNA (mRNA), which results in prevention of mRNA translation or degradation. ${ }^{9} 10$ A decrease in miRNAs can result in the overexpression of target genes. Previous studies have shown that 98 miRNAs are located at genomic regions involved in human cancers, indicating that miRNAs are required for the development of cancers. ${ }^{11}$ MiRNAs also play critical roles in many cellular processes in carcinogenesis and progression, including tumor growth, ${ }^{12}$ metastasis, ${ }^{13}$ and apoptosis, ${ }^{14}$ and can act as either tumor suppressors or oncogenes. ${ }^{15}$

MiR-497 was first identified in 2009 as a predictive biomarker of patient survival in neuroblastoma. ${ }^{16}$ The expression level of miR-497 was significantly decreased in various cancers, ${ }^{17,18}$ and has been reported to play as a tumor suppressor in a variety of cancers. ${ }^{19,20}$ Human miRNA microarray studies have shown that the expression level of miR-497 was significantly downregulated in human osteosarcoma. ${ }^{21}$ However, the exact role of miR-497 in evolution and progression of osteosarcoma remains unclear.

The membrane-associated protein angiomotin is an angiostatin-binding protein encoded by the $A M O T$ gene and was first described in $2001 .{ }^{22}$ Studies have shown that $A M O T$ gene expression and angiomotin is an important feature of breast cancer and plays an important role in endothelial cell migration and angiogenesis. ${ }^{23,24}$ At present, more than $30 \%$ of all human genes, as well as cellular processes, have been regulated or controlled by miRNAs. ${ }^{25}$ The increasing evidence showed the crucial impact of miRNA on occurrence and development of human cancers. ${ }^{26-28}$ Dysregulation of miRNAs plays important roles in cancer cell growth, ${ }^{28}$ cell apoptosis, ${ }^{29}$ and cell invasion. ${ }^{30}$ However, the role of $A M O T$ and angiomotin in the development and progression of osteosarcoma remains poorly understood.

We have recently used the approach of studying human osteosarcoma tissues and osteosarcoma cell lines to demonstrate that the long noncoding RNA SNHG12 promotes tumor cell proliferation and migration by upregulating of AMOT gene expression..$^{31}$ In this preliminary study, a similar methodology approach was chosen, using histologically confirmed human osteosarcoma tissue, normal tissue, and osteosarcoma cell lines and normal mesenchymal cell lines. The aim of this study was to determine whether miR-497 has a role in tumor suppression in human osteosarcoma.

\section{Materials and methods Compliance with ethical standards}

The protocol for this study was approved by the Research Ethics Committee of the Tianjin Medical University General Hospital. All patients agreed to participate in this study and provided a signed informed consent before enrollment.

\section{Tissues and cell lines}

Human primary osteosarcoma tissues $(\mathrm{n}=20)$ and matched adjacent normal tissues $(n=20)$ were obtained from 20 patients (13 males and seven females; $17.6 \pm 6.8$ years of age) attending the Department of General Surgery, Tianjin Medical University General Hospital, from 2012 to 2014. All the patients gave informed consent to participate in the study. All patients had a histological diagnosis of primary osteosarcoma according to the clinicopathological criteria of the International Union for Cancer Control. Histological assessment of tissue samples confirmed that each osteosarcoma sample contained $>70 \%$ tumor cells. Patient consents the use of tissue specimens in this research study, and the study protocols were approved by the Research Ethics Committee of Tianjin Medical University General Hospital.

Human osteosarcoma cell lines (SAOS-2, MG-63, U-2 osteosarcoma) and human osteoblasts cell line hFOB (OB3) were purchased from the American Type Culture Collection (ATCC) (Manassas, VA, USA). The osteosarcoma and osteoblast cells were maintained in culture according to the vendor's instructions. In brief, SAOS-2 and U-2 osteosarcoma cells were cultured in McCoy's 5a Medium (Modified) (ATCC). MG-63 cells were cultured in Eagle's Minimum Essential Medium (EMEM) (ATCC), while hFOB (OB3) cells were cultured in a 1:1 mixture of Ham's F12 Medium Dulbecco's Modified Eagle's Medium, with $2.5 \mathrm{mM}$ L-glutamine (without phenol red). All cells were cultured in media containing $10 \%$ fetal bovine serum (FBS) and $1 \%$ penicillin-streptomycin $(100 \mathrm{U} / \mathrm{mL}$ penicillin and $100 \mu \mathrm{g} / \mathrm{mL}$ streptomycin) and maintained in a humidified incubator under standard conditions.

\section{Quantitative real-time polymerase chain reaction}

Trizol reagent (Invitrogen Inc., Waltham, MA, USA) was used to extract total RNA from tissue samples or cultured cells. The relative expression of miR-497 was detected using a SYBR PrimeScript miRNA quantitative real-time polymerase chain reaction Kit (TAKARA, Dalian, People's Republic of China) in accordance with the manufacturer's instructions, and U-6 was used as an internal control. Approximately $2 \mu \mathrm{g}$ of total RNA was used to synthesize 
complementary DNA using Moloney Murine Leukemia Virus Reverse Transcriptase (Promega Corporation, Fitchburg, WI, USA), and subjected to quantitative real-time PCR reaction conditions: $95^{\circ} \mathrm{C}, 5$ minutes; $95^{\circ} \mathrm{C}, 10$ seconds; $60^{\circ} \mathrm{C}, 20$ seconds, 40 cycles. Gene expression was normalized to the level of GAPDH within each sample using the relative $\Delta \Delta \mathrm{CT}$ method. The sequence-specific primers were synthesized by Sangon (Shanghai, People's Republic of China) (Table 1). The quantitative real-time PCR was performed using the Applied Biosystems 7500 Sequence Detection System (ABI, Vernon, CA, USA).

\section{Western blot}

Total proteins were extracted using sodium dodecyl sulfate lysis buffer (Beyotime, Jiangsu, People's Republic of China), and bicinchoninic acid protein assay kit (Pierce, Rockford, IL, USA) was used to determine the concentration of total proteins. Equal amounts of total proteins were separated on $12 \%$ sodium dodecyl sulfate-polyacrylamide gels and transferred to polyvinylidene difluoride membranes (Millipore Corporation, Billerica, MA, USA) in $25 \mathrm{mM}$ Tris base and $190 \mathrm{mM}$ glycine at $50 \mathrm{~V}$ for 3 hours at $4^{\circ} \mathrm{C}$. The membranes were subjected to 2 hours blocking in Tris-buffered saline containing $5 \%$ nonfat dried milk powder at $37^{\circ} \mathrm{C}$ and incubated with primary antibody (angiomotin, 1:8,000, Abcam, Cambridge, UK; GAPDH, 1:2,000, Abcam) for 1 hour at $37^{\circ} \mathrm{C}$. The membranes were washed and subsequently treated with secondary antibody, goat anti-rabbit IgG conjugated to horseradish peroxidase (HRP) (Santa Cruz Biotechnology, Santa Cruz, CA, USA) at a 1/4,000 dilution for 1 hour at room temperature and visualized by chemiluminescence.

\section{Plasmid construction}

AMOT 3'-untranslated region (3'-UTR) containing putative binding sites for miR-497 was amplified from normal human genome DNA and cloned downstream of the psi-CHECK2 vector (Promega Corporation, Madison, WI, USA), identified as $A M O T-3^{\prime}$ UTR-WT. AMOT mutant 3'-UTR recombinant plasmid was generated using the QuikChange Site-Directed Mutagenesis Kit (Stratagene, La Jolla, CA, USA), which generated a mutation of $7 \mathrm{bps}$ from UGCUGCU to CAGGTAC in the predicted miR-497 target binding site, identified as AMOT-3'UTR-MUT.

\section{Luciferase assays}

For the reporter assay, the MG-63 cells were cultured in 24 -well plates 1 day before transfection. Approximately $50 \mathrm{ng}$ of $A M O T-3^{\prime} \mathrm{UTR}-\mathrm{WT}$ or -MUT vectors were cotransfected with $100 \mathrm{nM}$ miR-497 mimics or negative control (NC) into MG-63 cells using Lipofectamine 2000 reagent (Invitrogen), as described previously. After 48 hours of transfection, luciferase activity was measured using the Dual-Luciferase Reporter Assay System (Promega Corporation) according to the manufacturer's instructions. Firefly luciferase activity was normalized to the activity of Renilla luciferase.

\section{Transfection}

The miR-497 mimics, miR-497 inhibitor, and the scrambled sequence pre-miRNA NC were purchased from a commercial manufacturer (Ribobio, Guangzhou, People's Republic of China). Small interfering RNA (siRNA) targeting the $A M O T$ gene was obtained from Santa Cruz Biotechnology. Approximately $1 \times 10^{5}$ cells per well were seeded in 96-well plates and incubated for 24 hours, then cells were transfected with miR-497 mimics, miR-497 inhibitor, scrambled sequence (NC), or AMOT-siRNA (100 nM) using Lipofectamine 2000 (Invitrogen) in serum-free medium in accordance with the manufacturer's instructions.

\section{Cell proliferation assay}

Cell proliferation of MG-63 with mimics or siRNA duplexes was determined using the Cell Counting Kit 8 (CCK-8)

Table I Primer sequences used for miRNA and mRNA expression analysis

\begin{tabular}{ll}
\hline Name & Primer sequence $\left(\mathbf{5}^{\prime} \mathbf{-} \mathbf{3}^{\prime} \mathbf{)}\right.$ \\
\hline miR-497-RT & CTCAACTGGTGTCGTGGAGTCGGCAATTCAGTTGAGACAAACCA \\
U6-RT & CGCTTCACGAATTGGGTGTCAT \\
U6-F & CTCGCTTCGGCAGCACA \\
U6-R & AACGCTTCACGAATTTGCGT \\
miR-497-F & ACACTCCAGCTGGGCAGCAGCACACT \\
Universal-R & TGGTGTCGTGGAGTCG \\
AMOT-F & TAGGCATTCTCCTGGGTGGA \\
AMOT-R & GATTCCGTCCCACGTTCAGT \\
GAPDH-F & ACACCCACTCCTCCACCTTT \\
GAPDH-R & TTACTCCTTGGAGGCCATGT \\
\hline
\end{tabular}

Abbreviations: $F$, forward primer; R, reverse primer; RT, reverse transcription primer; miRNA, microRNA; mRNA, messenger RNA. 
(Dojindo Laboratories, Kumamoto, Japan) in accordance with the manufacturer's instructions. Briefly, $100 \mu \mathrm{L}$ of exponentially growing MG-63 cells $\left(1 \times 10^{5}\right.$ cells $\left./ \mathrm{mL}\right)$ were cultured in 96-well culture plates with $10 \%$ FBS for 0,24 , 48,72 , and 96 hours. Then, $10 \mu \mathrm{L}$ of the CCK-8 solution was added to each well and incubated at $37^{\circ} \mathrm{C}$ for 2 hours. The optical density values were recorded using a Thermo-plate microplate reader (Rayto Life and Analytical Science Co. Ltd, Germany) at $450 \mathrm{~nm}$.

\section{Flow cytometry}

Cell apoptosis was determined using an Annexin-V fluorescein isothiocyanate and propidium iodide (FITC/PI) apoptosis detection kit (Life Technologies, Waltham, MA, USA) with flow cytometry in accordance with the manufacturer's instruction. Briefly, $1 \times 10^{6}$ cells were harvested, resuspended, and then stained using Annexin-V FITC/PI. Cell samples were analyzed using flow cytometry and a FACScan (Becton-Dickinson, Franklin Lakes, NJ, USA). Annexin-V(+)/PI(-) and Annexin-V(+)/PI(+) represented the MG-63 cells in early apoptosis and late apoptosis/necrosis, respectively.

\section{Migration and invasion assays}

The 24-well transwell chambers (Millipore Corporation) were used for the cell invasion assay in accordance with the manufacturer's protocol. Briefly, $1 \times 10^{5}$ cells in serum-free EMEM were added to the upper chamber of the Matrigelcoated insert, and $500 \mu \mathrm{L}$ of EMEM containing 10\% FBS was added to the lower chamber for culture. The cells were cultured in a humidified atmosphere for 24 hours at $37^{\circ} \mathrm{C}$, and $5 \% \mathrm{CO}_{2}$, and the noninvading cells were removed. The cells were then fixed with $100 \%$ methanol for 30 minutes and stained using $0.5 \%$ crystal violet (Sigma-Aldrich, St Louis, MO, USA) for 20 minutes. The migrating cells were counted under a phase-contrast microscope (Olympus, Tokyo, Japan). Migration assays were applied similarly without coating the upper chamber with Matrigel, and migrating cells were counted at 24 hours.

\section{Statistical analysis}

All experiments and tests on cell and tissue samples were performed in triplicate. All numerical results were expressed as the mean \pm standard deviation. Statistical significance between groups was determined using one-way analysis of variance or a paired or an unpaired Student's $t$-test was performed using SPSS 20.0 (IBM SPSS, Chicago, IL, USA). To assess the interaction between the variables, the miR-497 expression levels were cross-correlated with $A M O T$ gene expression in the univariate Cox model; the interaction was evaluated by the Wald test. A $P$-value of $<0.05$ was considered to be statistically significant.

\section{Results}

\section{Angiomotin expression in osteosarcoma tissues}

To investigate the expression level of angiomotin in osteosarcoma, Western blot was used to measure the angiomotin levels in osteosarcoma tissues and matched adjacent normal tissues. As shown in Figure 1, the expression level of angiomotin was significantly increased in tumor tissues compared with matched normal tissues (Figure 1A), and the relative expression of angiomotin in tumor tissues and normal tissues was $1.36 \pm 0.32$ fold-change and $3.86 \pm 0.69$ fold-change, respectively $(P<0.001)$ (Figure $1 \mathrm{~B})$. These results suggest that the $A M O T$ gene and its protein product, angiomotin, may play a role in human osteosarcoma.

\section{MiR-497 downregulation in osteosarcoma tissue and its negative correlation with angiomotin expression}

The expression of miR-497 was analyzed by quantitative realtime PCR. The miR-497 expression levels (Figure 1C) were decreased in osteosarcoma tissue, compared with patientmatched normal tissue. Angiomotin protein expression levels were inversely correlated with miR-497 expression levels in the 20 osteosarcoma tissues studied $(P=0.0001 ; R=0.7652$; Figure 1D).

\section{Overexpression of miR-497 suppressed cell proliferation in vitro}

The expression of angiomotin protein in osteosarcoma cells was analyzed using Western blot. The angiomotin protein levels (Figure 2A) were greatest in the MG-63 cells, with the relative expression levels of angiomotin being $3.82 \pm 0.34$ compared with the OB3 cell line (Figure 2B). In addition, miR-497 was downregulated in MG-63 cells, with the relative expression level being $0.36 \pm 0.28$ (Figure $2 \mathrm{C}$ ). Following this result, MG-63 cells were selected for further study.

To evaluate the role of miR-497 on cell proliferation, miR-497 was overexpressed in MG-63 cells by transfection with miR-497 mimics. Following transfection, the relative expression of miR-497 was increased 71.62-fold (Figure 2D). The proliferation of miR-497-transfected cells was significantly suppressed compared with the NC-transfected MG-63 cells ( $P<0.05$ at 48, 72, and 96 hours) (Figure 2E). 
A

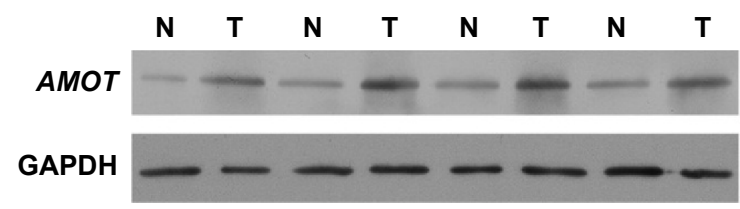

C

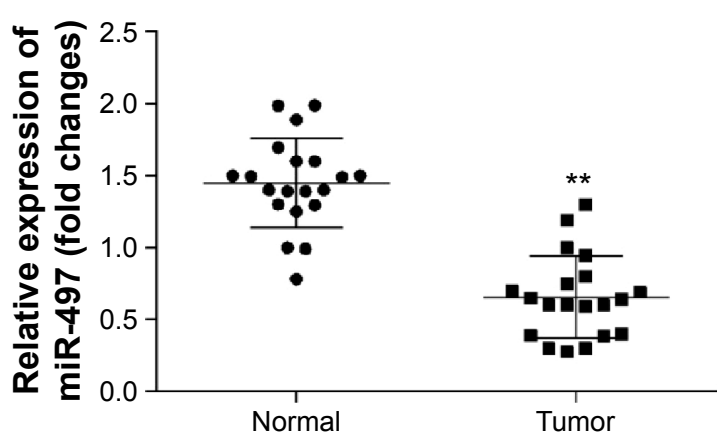

B

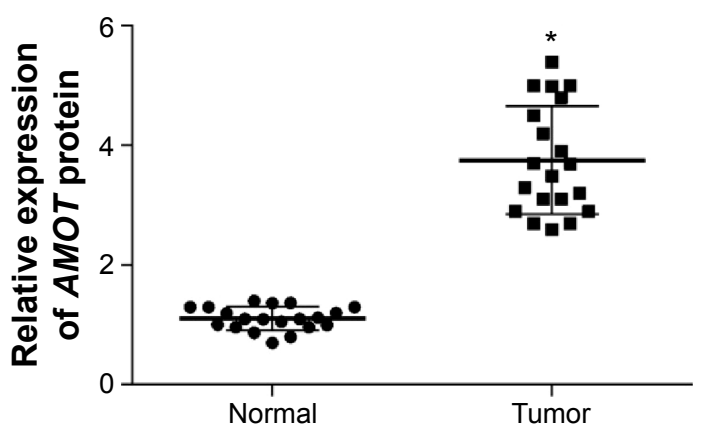

D

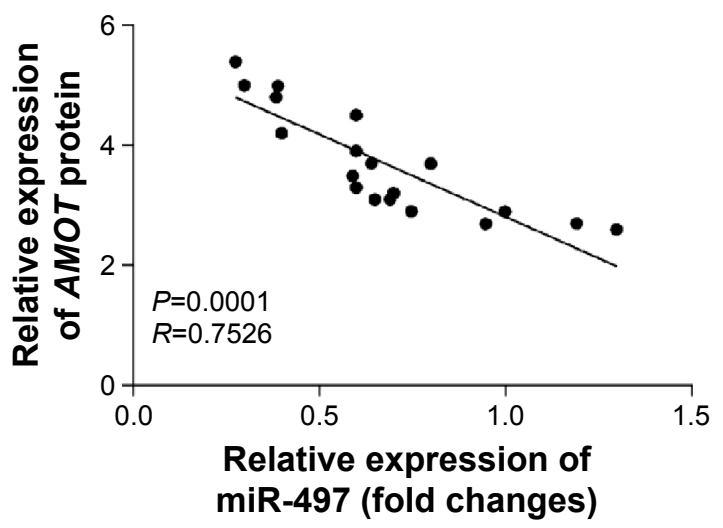

Figure I Expression of miR-497 and angiomotin, the protein product of the AMOT gene, in osteosarcoma tissues.

Notes: (A) Western blot analysis of angiomotin expression in both T and N. (B) Quantification of the protein bands (OD ratio over GAPDH), *P $<0.00 \mathrm{I}$. (C) The expression levels of miR-497 in osteosarcoma samples and normal tissues were quantified by quantitative real-time PCR; the miR-497 was significantly downregulated in tumor samples. $* * P<0.0$ I. (D) Angiomotin protein expression levels were inversely correlated with miR-497 expression levels $(P=0.000 \mathrm{I}, R=0.7562)$.

Abbreviations: $\mathrm{T}$, tumor samples; $\mathrm{N}$, normal tissues; OD, odds.

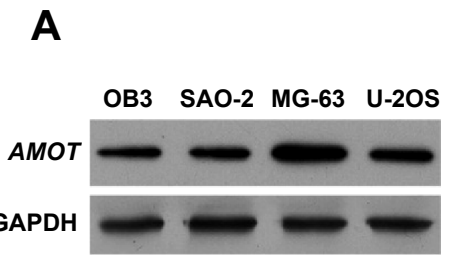

D

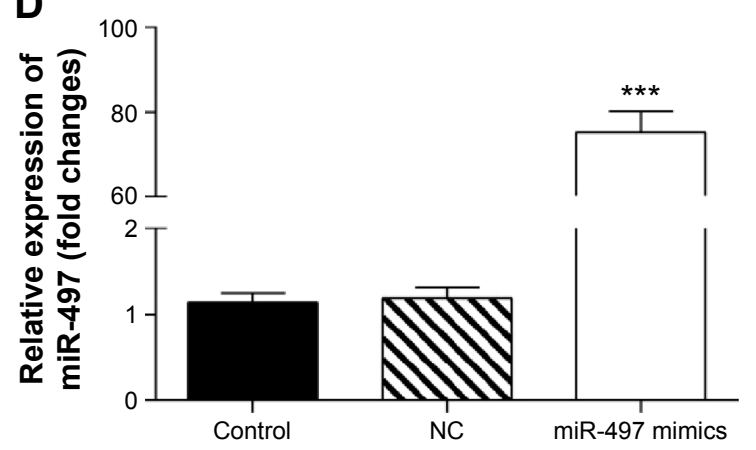

B

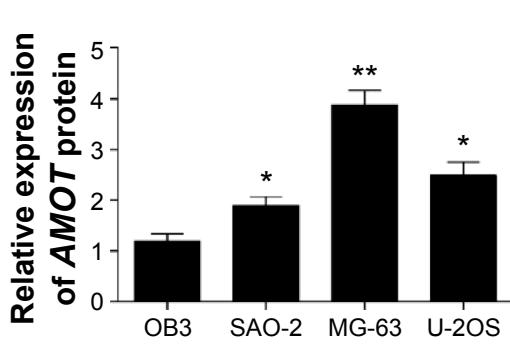

C

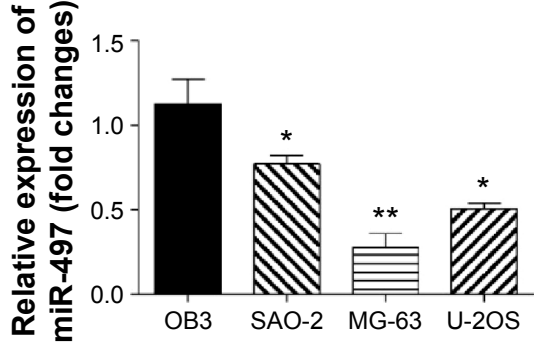

E

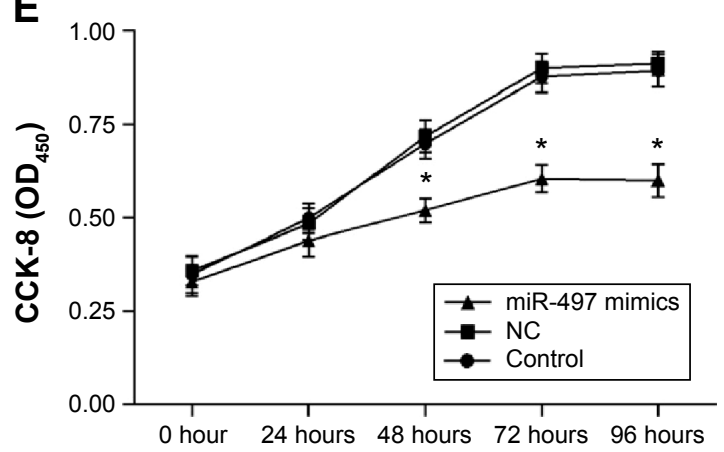

Figure 2 Overexpression of miR-497 inhibited cell proliferation.

Notes: (A) The expression level of angiomotin, the protein product of the AMOT gene, in OB3, SAO-2, MG-63, and U-2OS cells was detected using Western blot. (B) Quantification of the protein bands (OD ratio over GAPDH). (C) The expression level of miR-497 in OB3, SAO-2, MG-63, and U-2OS cells was detected using quantitative real-time PCR. (D) The expression level of miR-497 was detected using quantitative real-time PCR following transfection with miR-497 mimics. (E) CCK-8 assay results showed that the MG-63 cell proliferation was inhibited following transfection with miR-497 mimics. $* P<0.05 ; * * P<0.01 ; * * * P<0.001$.

Abbreviations: OD, odds; CCK-8, Cell Counting Kit 8; NC, negative control. 


\section{Overexpression of miR-497 suppressed} cell migration and invasion in vitro

The role of miR-497 in tumor cell migration and invasion was detected using transwell assay. The cell migration and invasion of MG-63 were inhibited by transfection with miR497-mimics $(P<0.01)$ (Figure 3A and B).

\section{Overexpression of miR-497 promoted osteosarcoma cell apoptosis}

The Annexin-VFITC/PI staining method to detect cell apoptosis demonstrated that the cell apoptosis rate was increased following transfection with the miR-497 mimics $(P<0.01)$ (Figure 4A). The apoptosis rates of MG-63 cells and transfected MG-63 cells compared with NC were $7.2 \pm 0.54 \%$ and $7.51 \pm 0.63 \%$, respectively; the apoptosis rate of $\mathrm{MG}-63$ cells transfected with miR-497 mimics was 19.4 $\pm 1.32 \%$ (Figure 4B).

\section{Identification of AMOT as the target gene of miR-497}

The expression of angiomotin, the protein product of the AMOT gene, was determined in MG-63 cells following transfection with miR-497 mimics using Western blot (Figure 5A). In addition, miR-497 target-predictive databases (TargetScan and miRDB) were used for computational analyses. MiR-497 has one predictive target site in humans, AMOT $3{ }^{\prime}$-UTR (Figure 5B). A dual-luciferase reporter system was employed to verify whether the $A M O T$ gene is a direct target of miR497. The results showed that the miR-497 mimics downregulated the luciferase activity of the reporter and that the luciferase expression of mutant 3'-UTR of AMOT was no longer subject to regulation by miR-497 (Figure 5C). These results indicated that this site in the $3^{\prime}$-UTR of AMOT was the specific regulation site for miR-497.

\section{Knockdown of the AMOT gene suppressed the proliferation of MG-63 cells}

To explain the decreased expression of angiomotin in $A M O T$-siRNA transfected MG-63 cells, the expression of $A M O T$ mRNA and protein levels were analyzed by quantitative real-time PCR and Western blot, respectively. The angiomotin protein expression level (Figure 6A and B) and mRNA expression (Figure 6C) of AMOT were significantly decreased in MG-63 cells transfected with AMOTsiRNA. The results showed downregulation of $A M O T$ in the $A M O T$-siRNA transfected MG-63 cells. The CCK-8 assay showed that the knockdown of the AMOT gene inhibited cell proliferation (Figure 6D).

\section{Knockdown of the AMOT gene suppressed cell migration and invasion in vitro}

The role of $A M O T$ on tumor cell migration and invasion was investigated using a transwell assay. The cell migration and
A
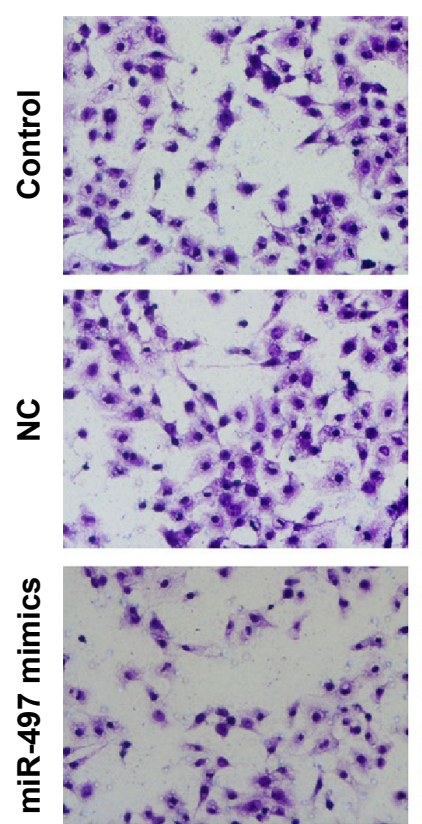

Migration

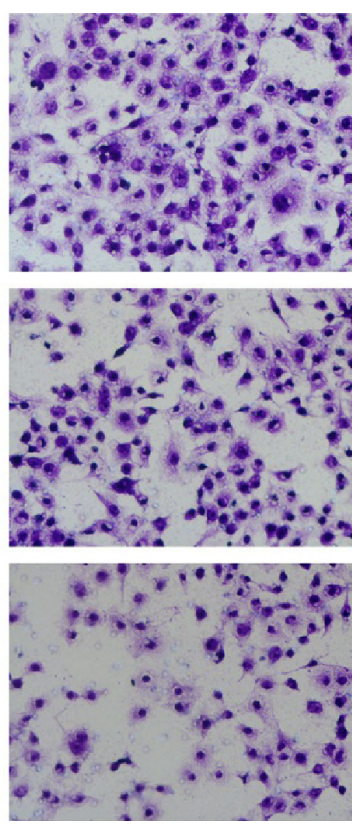

B
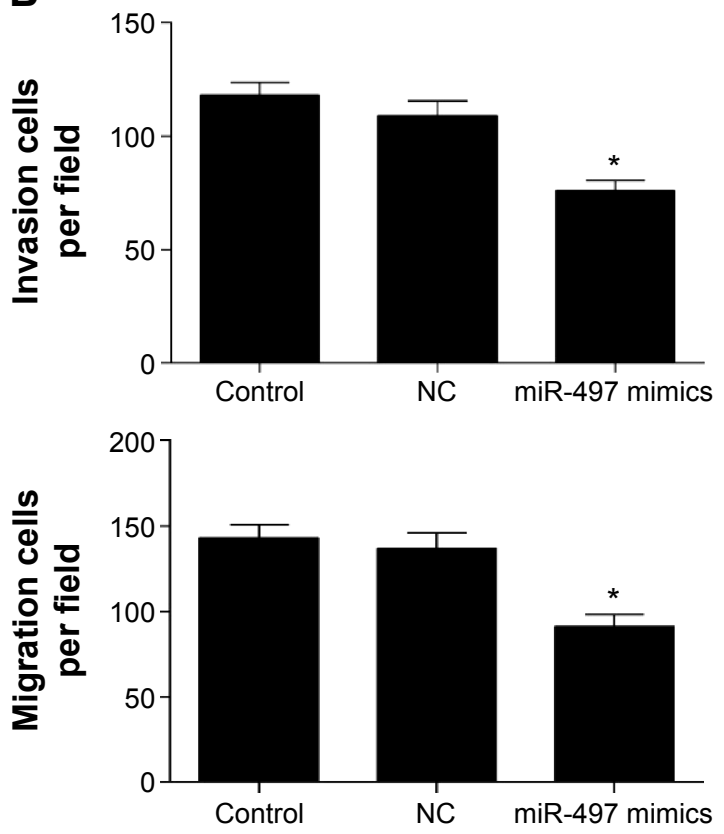

Figure 3 Overexpression of miR-497 inhibited cell migration and invasion.

Notes: (A) Transwell assay was used to detect cell migration and invasion ( $\times 40$ magnification). Images of migration and invasion of each cell group are presented. (B) The average migration and invasion cell number per field among different experimental groups. Data are the mean \pm SD from three independent experiments, $* P<0.0 \mathrm{I}$.

Abbreviations: SD, standard deviation; NC, negative control. 

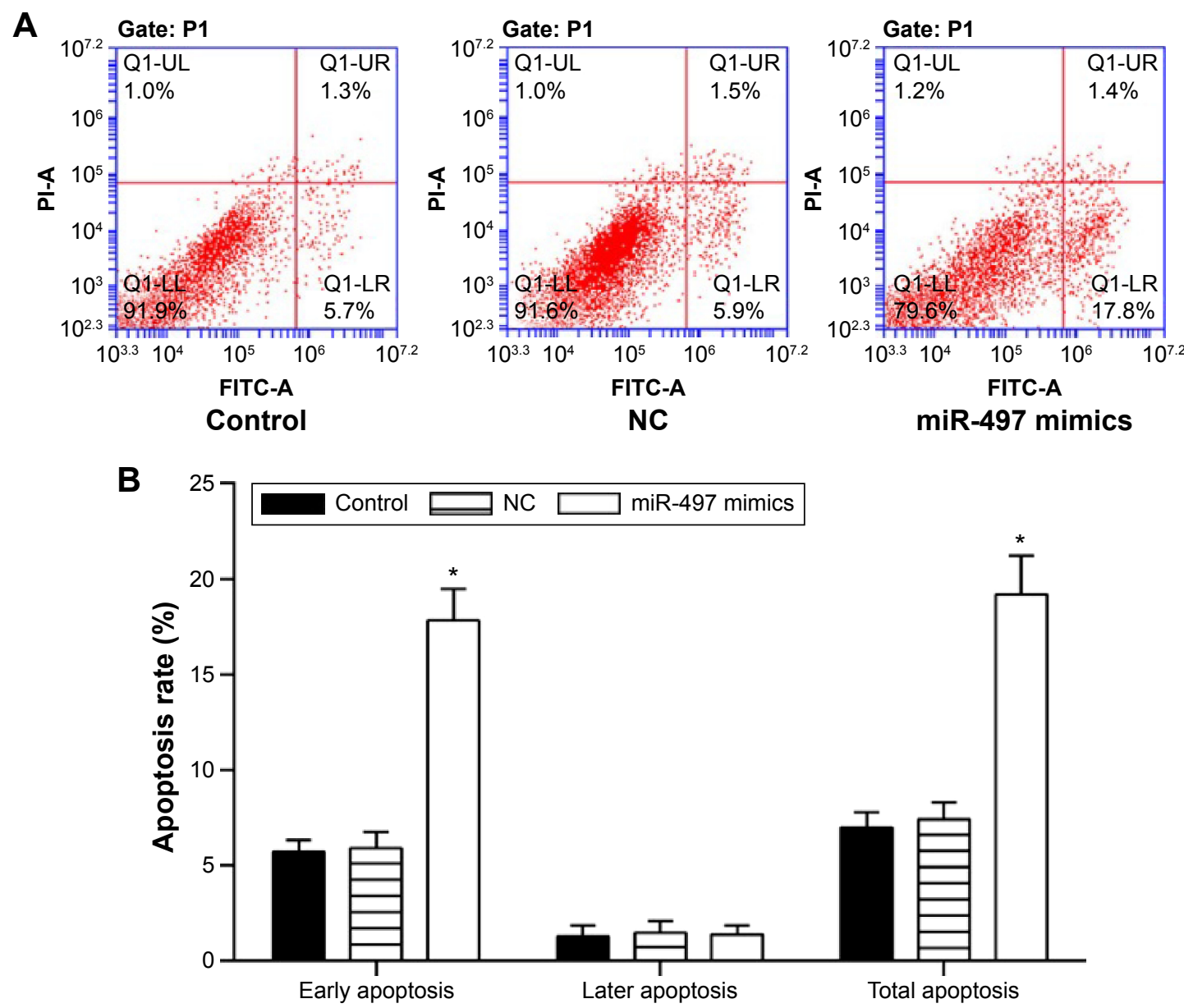

Figure 4 Overexpression of miR-497 promoted osteosarcoma cell apoptosis.

Notes: Cell death was monitored by Annexin-V FITC/PI staining and flow cytometry. Quadrant statistics: early apoptotic cells in LR and late apoptotic cells in UR; viable cells and necrotic cells shown separately in LL and UL. (A) Flow cytometry analysis of MG-63 cells in different experimental groups. (B) The percentage of early apoptotic cells, late apoptotic cells, and total apoptotic cells of each group. $* P<0.01$. Data are representative of three experiments and are expressed as mean \pm SD.

Abbreviations: FITC/PI, fluorescein isothiocyanate and propidium iodide; LR, lower right; UR, upper right; LL, lower left; UL, upper left; SD, standard deviation; NC, negative control.

invasion of MG-63 cells was inhibited by knockdown of AMOT $(P<0.01)$ (Figure 7A and $\mathrm{B})$. In contrast, the suppression of $A M O T$ had no effect on MG-63 cell apoptosis (Figure 8).

\section{Discussion}

In this preliminary study on human osteosarcoma, miR-497 was found to be significantly downregulated in osteosarcoma tissues and cell lines when compared with normal tissues and cells, indicating a potential tumor suppressor role for miR-497 in osteosarcoma. The role of miR-497 in osteosarcoma cell growth, migration, and apoptosis was studied using a variety of methods, including flow cytometry and apoptosis analysis and migration and invasion assays. In addition, transfection of miR-497 mimics inhibited osteosarcoma cell proliferation, migration, and invasion and promoted cell apoptosis in vitro in the MG-63 cell line. These findings support a role for miR-497 in the invasive and metastatic potential of osteosarcoma. Although this is a small and preliminary study, the findings would support further laboratory and clinical studies, particularly as there is a requirement for diagnostic and predictive biomarkers in osteosarcoma.

The findings of the present study support the hypothesis that the AMOT gene is a direct target of miR-497 in human osteosarcoma. In this study, AMOT expression levels were increased in human osteosarcoma tumor cells when compared with patient-matched normal tissues; levels of the AMOT protein product, angiomotin, were inversely correlated with miR-497 expression. Knockdown of the AMOT gene inhibited MG-63 cell proliferation and migration, but it did not affect cell apoptosis. Overexpression of miR-497 significantly decreased the expression of angiomotin by binding the complementary sites of the AMOT gene 3'-UTR. Angiomotin, the protein product of $A M O T$, is a membraneassociated protein that binds to the endothelial cell surface 
A

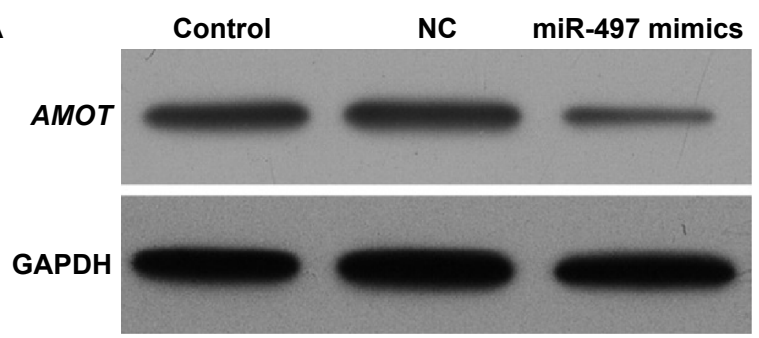

B

3' UGUUUGGUGUCACACGACGAC 5' miR-497

| | | | | | |

2944: 5' GAUUUCCUUUUAAUGCUGCUU 3' AMOT-WT

2944: 5' GAUUUCCUUUUAACAGGTACU 3' AMOT-MUT

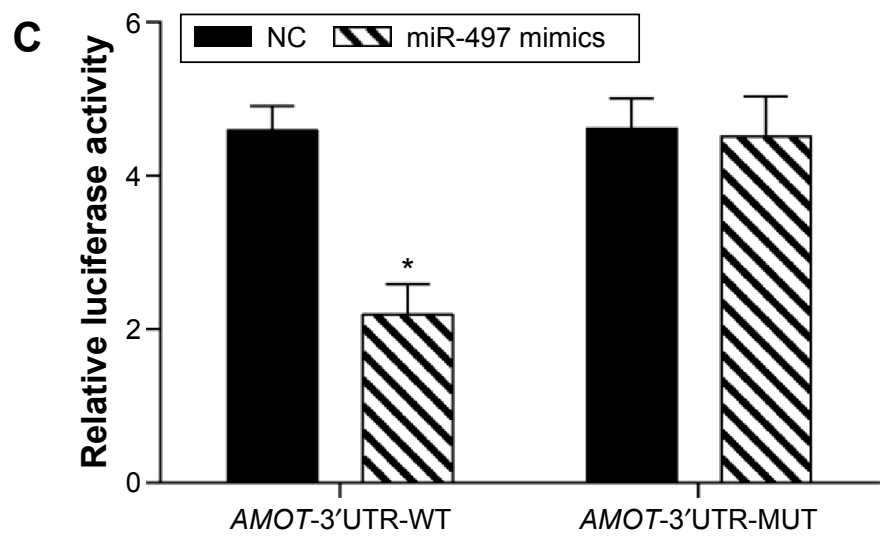

Figure 5 The AMOT gene, a target gene for miR-497.

Notes: (A) The expression level of angiomotin, the protein product of the AMOT gene in MG-63 cells following transfection with miR-497 mimics. (B) Sequence alignment of miR-497 and 3'-UTR of AMOT using the TargetScan and miRDB algorithms. (C) The MG-63 cells were cotransfected with miR-497 mimics and a luciferase reporter containing a fragment of the AMOT $3^{\prime}$-UTR harboring either the miR-497 binding site (AMOT-3'-UTR-WT) or a mutant (AMOT-3'-UTR-MUT). The assay showed that luciferase activity in the AMOT-3'-UTR-WT group was significantly decreased but was increased when compared with the luciferase activity of the mutant groups. $* P<0.05$. Abbreviations: UTR, untranslated region; NC, negative control.

A

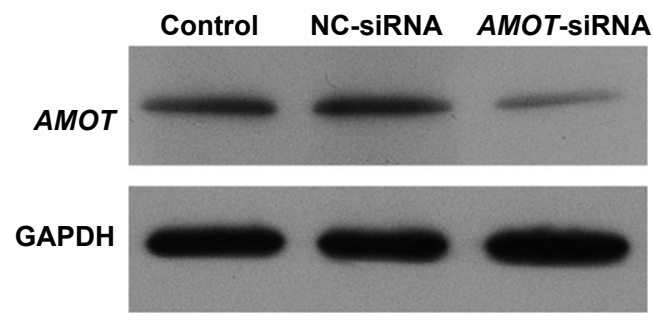

C

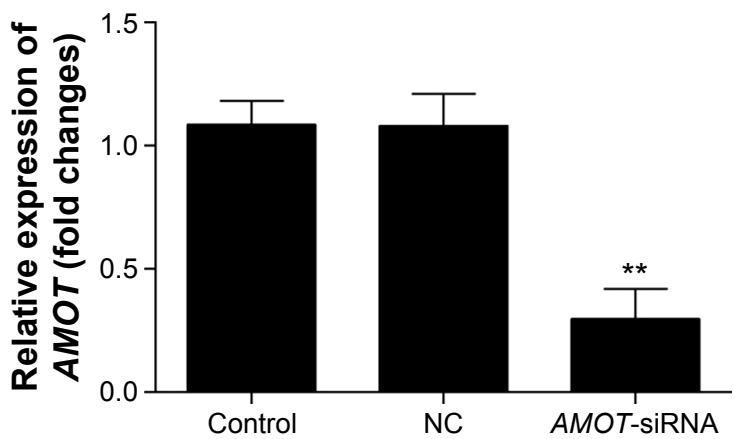

B

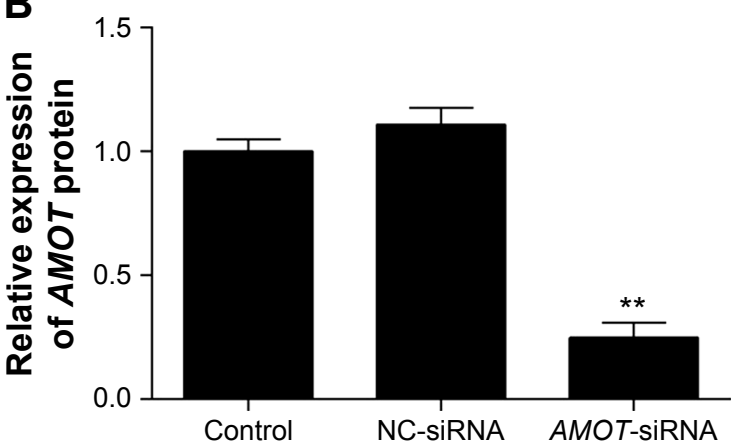

D

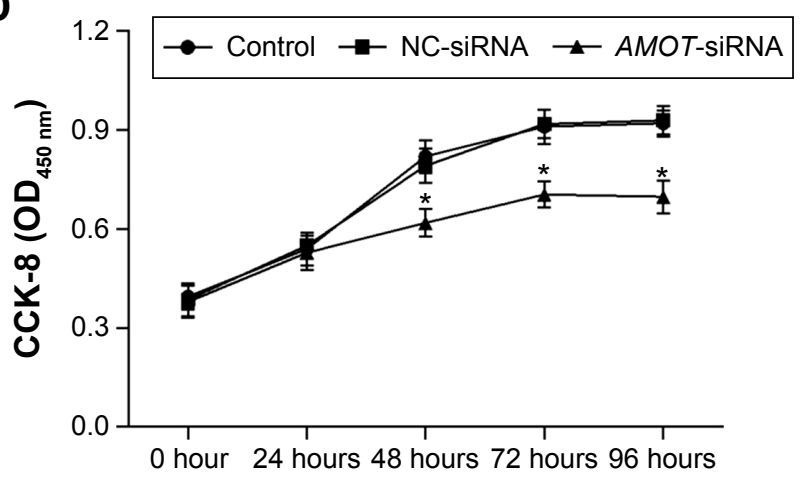

Figure 6 Knockdown of AMOT inhibited MG-63 cell proliferation.

Notes: (A) The expression levels of angiomotin, the protein product of the AMOT gene in MG-63 cells transfected with AMOT-siRNA and scramble NC siRNA was determined using Western blot. (B) Quantification of the angiomotin protein bands (OD ratio over GAPDH), **P $<0.0$ I. (C) quantitative real-time PCR was used to detect the expression of $A M O T$ in MG-63 cells transfected with AMOT-siRNA and in the NC. $* * P<0.01$. (D) The cell proliferation was suppressed following knockdown of $A M O T$ gene expression. $* P<0.01$.

Abbreviations: siRNA, small interfering RNA; NC, negative control; OD, odds; CCK-8, Cell Counting Kit 8. 

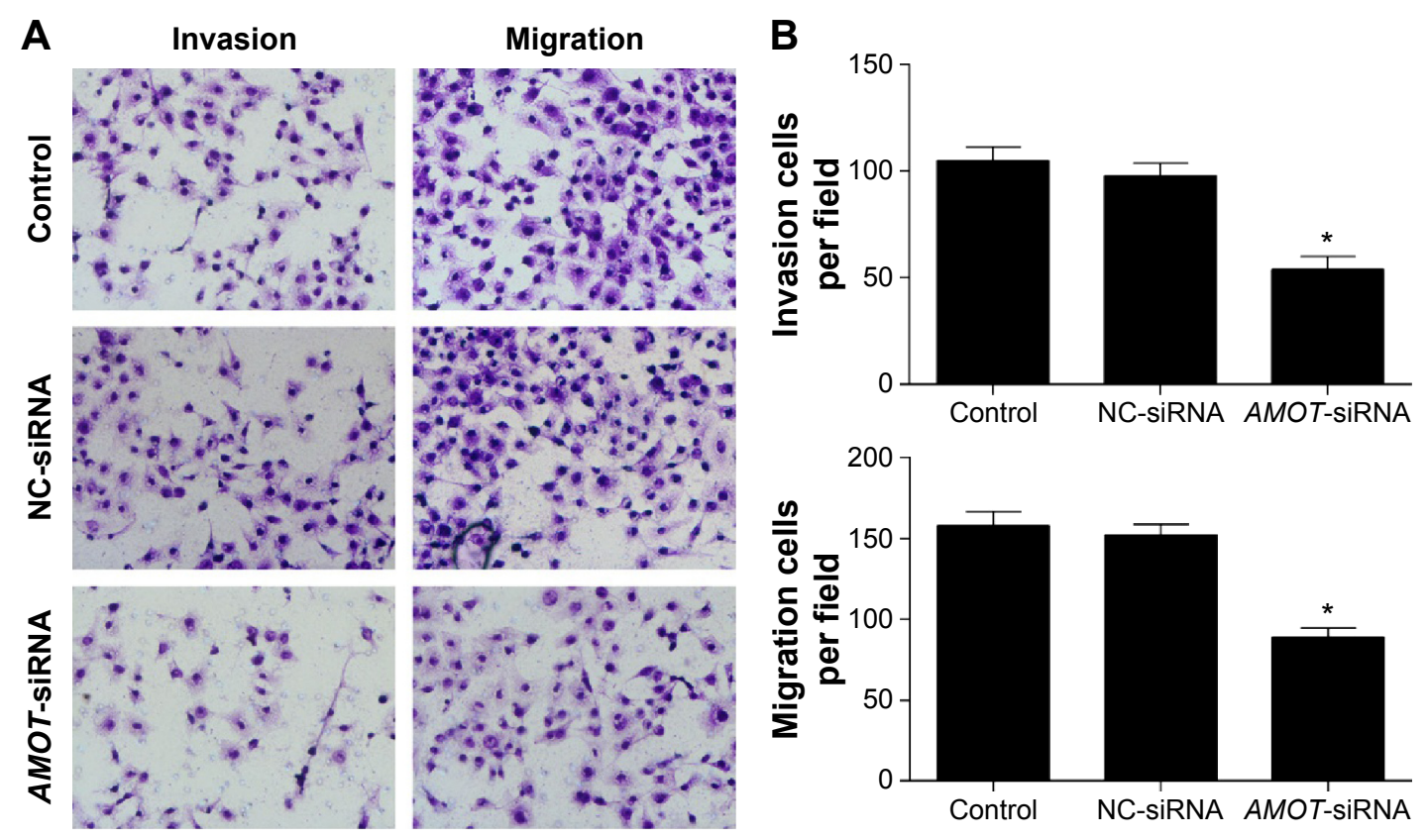

Figure 7 Knockdown of AMOT inhibited MG-63 cell migration and invasion.

Notes: (A) Transwell assay was used to examine cell migration and invasion at 48 hours following AMOT-siRNA or NC-siRNA transfection. Photomicrographs of the invasive ability of each cell group are presented ( $\times 40$ magnification). (B) Invasion and migration assay data are representative of three experiments and are expressed as mean \pm SD, $* P<0.0$ I. Abbreviations: siRNA, small interfering RNA; NC, negative control; SD, standard deviation.
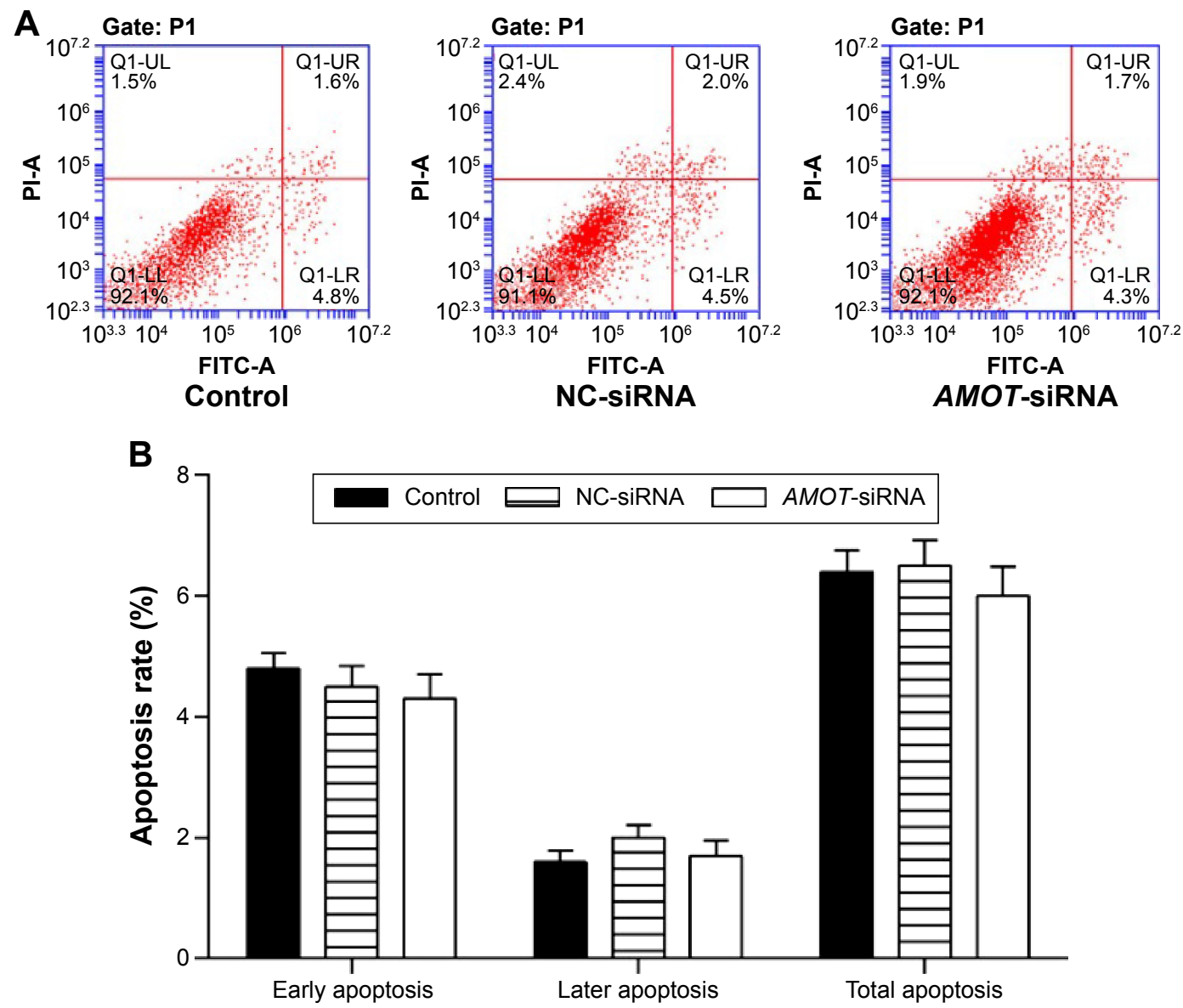

Figure 8 Knockdown of AMOT does not affect MG-63 cell apoptosis.

Notes: (A) Cell apoptosis was detected following AMOT-siRNA or NC-siRNA transfection at 48 hours. (B) The percentage of cells in different stages of apoptosis is displayed in histograms and as mean \pm SD.

Abbreviations: siRNA, small interfering RNA; NC, negative control; SD, standard deviation; FITC, fluorescein isothiocyanate; PI, propidium iodide; UL, upper left; UR, upper right; LL, lower left; LR, lower right. 
of angiogenic cells that are involved in controlling cell migration. ${ }^{32}$ A previous study has shown that AMOT can activate ERK1/2 protein kinase pathway through regulating Rac1 GTPase directly, which enhances cell migration and stabilizes angiogenic blood vessels. ${ }^{33}$ Furthermore, AMOT has been reported to be overexpressed in human cancers and to play an important role in inhibiting the growth of tumors and altering tumor vessel permeability. ${ }^{24,34}$ These previously published findings are consistent with the findings of the present study that overexpression of miR-497 suppresses the proliferation and migration of human osteosarcoma cells.

In this study, there was no significant difference in the mRNA expression of AMOT following miR-497 mimics transfection (data not shown), suggesting that miR-497 may regulate $A M O T$ expression and prevent mRNA from being translated. It is clear that further studies are required to determine the molecular mechanisms involved in the regulation of the AMOT gene by miR-497. This study was limited as it included a relatively small number of osteosarcoma tumor samples from a single center and as only a limited number of cell lines were studied. However, the method of studying human tumor samples with normal tissues and tumor cell lines with normal cell lines is, we believe, a valuable approach to understanding human tumor biology and is also a method that can be developed. ${ }^{31}$ In future studies, the role of miR-497 could be investigated by using osteosarcoma tissue taken from invasive tumor foci or metastatic deposits and comparing expression levels with those in the primary tumor. In addition, the preliminary in vitro studies reported here could be extended to include other human osteosarcoma cell lines.

\section{Conclusion}

These preliminary studies support a role for miR-497 as a suppressor of AMOT gene expression in human osteosarcoma cells, resulting in suppression of tumor cell proliferation and invasion. Further studies are recommended to investigate the role of miR-497 in osteosarcoma and other malignant mesenchymal tumors.

\section{Disclosure}

The authors report no conflicts of interest in this work.

\section{References}

1. Picci P. Osteosarcoma (osteogenic sarcoma). Orphanet J Rare Dis. 2007;2:6.

2. Walkley CR, Qudsi R, Sankaran VG, et al. Conditional mouse osteosarcoma, dependent on $\mathrm{p} 53$ loss and potentiated by loss of $\mathrm{Rb}$, mimics the human disease. Gene Dev. 2008;22(12):1662-1676.

3. Caudill JS, Arndt CA. Diagnosis and management of bone malignancy in adolescence. Adolesc Med State Art Rev. 2007;18(1):62-78, ix.
4. Huang J, Liu K, Yu Y, et al. Targeting HMGB1-mediated autophagy as a novel therapeutic strategy for osteosarcoma. Autophagy. 2012;8(2): 275-277.

5. Ando K, Heymann MF, Stresing V, Mori K, Rédini F, Heymann D. Current therapeutic strategies and novel approaches in osteosarcoma. Cancers. 2013;5(2):591-616.

6. Ottaviani G, Jaffe N. The epidemiology of osteosarcoma. Cancer Treat Res. 2009;152:3-13.

7. Tan ML, Choong PF, Dass CR. Osteosarcoma: conventional treatment vs gene therapy. Cancer Biol Ther. 2009;8(2):106-117.

8. Doench JG, Sharp PA. Specificity of microRNA target selection in translational repression. Gene Dev. 2004;18(5):504-511.

9. Bartel DP. MicroRNAs: genomics, biogenesis, mechanism, and function. Cell. 2004;116(2):281-297.

10. Filipowicz W, Bhattacharyya SN, Sonenberg N. Mechanisms of posttranscriptional regulation by microRNAs: are the answers in sight? Nat Rev Genet. 2008;9(2):102-114.

11. Calin GA, Sevignani C, Dumitru CD, et al. Human microRNA genes are frequently located at fragile sites and genomic regions involved in cancers. Proc Natl Acad Sci U S A. 2004;101(9):2999-3004.

12. Pramanik D, Campbell NR, Karikari C, et al. Restitution of tumor suppressor microRNAs using a systemic nanovector inhibits pancreatic cancer growth in mice. Mol Cancer Ther. 2011;10(8):1470-1480.

13. Bouyssou JMC, Manier S, Huynh D, Issa S, Roccaro AM, Ghobrial IM. Regulation of microRNAs in cancer metastasis. Biochim Biophys Acta. 2014;1845(2):255-265.

14. Di Leva G, Garofalo M, Croce CM. MicroRNAs in cancer. Аnпи Rev Pathol. 2014;9:287-314.

15. Lages E, Ipas H, Guttin A, Nesr H, Berger F, Issartel JP. MicroRNAs: molecular features and role in cancer. Front Biosci. 2012;17: 2508-2540.

16. Bray I, Bryan K, Prenter S, et al. Widespread dysregulation of MiRNAs by MYCN amplification and chromosomal imbalances in neuroblastoma: association of miRNA expression with survival. PLoS One. 2009; 4(11):e7850.

17. Zhao X, Zhao Z, Xu W, Hou J, Du X. Down-regulation of miR-497 is associated with poor prognosis in renal cancer. Int J Clin Exp Pathol. 2015;8(1):758-764.

18. Xu J, Wang T, Cao Z, et al. MiR-497 downregulation contributes to the malignancy of pancreatic cancer and associates with a poor prognosis. Oncotarget. 2014;5(16):6983-6993.

19. Du M, Shi D, Yuan L, et al. Circulating miR-497 and miR-663b in plasma are potential novel biomarkers for bladder cancer. Sci Rep. 2015;5: 10437.

20. Itesako $\mathrm{T}$, Seki $\mathrm{N}$, Yoshino $\mathrm{H}$, et al. The microRNA expression signature of bladder cancer by deep sequencing: the functional significance of the miR-195/497 cluster. PLoS One. 2014;9(2):e84311.

21. Namlos HM, Meza-Zepeda LA, Baroy T, et al. Modulation of the osteosarcoma expression phenotype by microRNAs. PLoS One. 2012;7(10): e48086.

22. Troyanovsky B, Levchenko T, Mansson G, Matvijenko O, Holmgren L. Angiomotin: an angiostatin binding protein that regulates endothelial cell migration and tube formation. J Cell Biol. 2001;152(6):1247-1254.

23. Holmgren L, Ambrosino E, Birot $\mathrm{O}$, et al. A DNA vaccine targeting angiomotin inhibits angiogenesis and suppresses tumor growth. Proc Natl Acad Sci U S A. 2006;103(24):9208-9213.

24. Yi C, Shen Z, Stemmer-Rachamimov A, et al. The p130 isoform of angiomotin is required for Yap-mediated hepatic epithelial cell proliferation and tumorigenesis. Sci Signal. 2013;6(291):ra77.

25. Nagadia R, Pandit P, Coman WB, Cooper-White J, Punyadeera C. MiRNAs in head and neck cancer revisited. Cell Oncol (Dordr). 2013; 36(1):1-7.

26. Wang YW, Shi DB, Chen X, Gao C, Gao P. Clinicopathological significance of microRNA-214 in gastric cancer and its effect on cell biological behaviour. PLoS One. 2014;9(3):e91307.

27. He H, Tian W, Chen H, Jiang K. MiR-944 functions as a novel oncogene and regulates the chemoresistance in breast cancer. Tumor Biol. Epub August 23, 2015. 
28. Li W, Zhang J, Chen T, Yin P, Yang J, Cao Y. miR-132 upregulation promotes gastric cancer cell growth through suppression of FoxO1 translation. Tumor Biol. Epub August 23, 2015.

29. Li C, Du X, Tai S, et al. GPC1 regulated by miR-96-5p, rather than miR-182-5p, in inhibition of pancreatic carcinoma cell proliferation. Int J Mol Sci. 2014;15(4):6314-6327.

30. Xue J, Chen Z, Gu X, Zhang Y, Zhang W. MicroRNA-148a inhibits migration of breast cancer cells by targeting MMP-13. Tumor Biol. Epub August 23, 2015.

31. Ruan W, Wuang P, Feng S, Xue Y, Li Y. Long non-coding RNA small nucleolar RNA host gene 12 (SNHG12) promotes cell proliferation and migration by upregulating angiomotin gene expression in human osteosarcoma cells. Tumour Biol. Epub October 10, 2015.
32. Levchenko T, Aase K, Troyanovsky B, Bratt A, Holmgren L. Loss of responsiveness to chemotactic factors by deletion of the $\mathrm{C}$-terminal protein interaction site of angiomotin. J Cell Sci. 2003;116(Pt 18): 3803-3810.

33. Yi C, Troutman S, Fera D, et al. A tight junction-associated Merlin-angiomotin complex mediates Merlin's regulation of mitogenic signaling and tumor suppressive functions. Cancer Cell. 2011; 19(4):527-540.

34. Arigoni M, Barutello G, Lanzardo S, et al. A vaccine targeting angiomotin induces an antibody response which alters tumor vessel permeability and hampers the growth of established tumors. Angiogenesis. 2012;15(2):305-316.

\section{Publish your work in this journal}

OncoTargets and Therapy is an international, peer-reviewed, open access journal focusing on the pathological basis of all cancers, potential targets for therapy and treatment protocols employed to improve the management of cancer patients. The journal also focuses on the impact of management programs and new therapeutic agents and protocols on

\section{Dovepress}

patient perspectives such as quality of life, adherence and satisfaction. The manuscript management system is completely online and includes a very quick and fair peer-review system, which is all easy to use. Visit http://www.dovepress.com/testimonials.php to read real quotes from published authors.

Submit your manuscript here: http://www.dovepress.com/oncotargets-and-therapy-journal 